\title{
Catheter ablation of outflow tract ventricular arrhythmia with intracardiac echocardiography assistance
}

\author{
Ji-Hoon Choi and Kyoung-Min Park *i)
}

\begin{abstract}
As the indications for catheter-based electrophysiologic procedures become more diverse and complex, accurate assessment of the anatomy of intracardiac structure has become essential for the optimal clinical outcome. Since intracardiac echocardiography (ICE) was first introduced in 1980, it has become an integral part of various electrophysiologic procedures enabling accurate visualization of cardiac structures and continuous monitoring of catheter position, with integration of real-time images and electroanatomic mapping. Catheter ablation for outflow tract ventricular arrhythmias (OTVAs) has been actively performed in symptomatic patients. However, the anatomic complexity of OT serves as the biggest obstacle to obtain the optimal results. In OTVAs, ICE has played an invaluable role in precisely defining the anatomic shell of OT beyond fluoroscopy and in guiding catheter manipulation in relation to critical structures like the conduction system and coronary arteries. This review article provides detailed information on comprehensive application of ICE for catheter ablation of OTVAs.
\end{abstract}

Keywords: Outflow tract, Intracardiac echocardiography, Catheter ablation, Ventricular arrhythmia

\section{Background}

The ventricular outflow tract $(\mathrm{OT})$ is the most common site of origin (SOO) for idiopathic ventricular arrhythmias (VAs) in patients without structural heart disease [1-3]. Multiple studies have been shown that radiofrequency catheter ablation (RFCA) can be successfully performed in OT with high success and low complication rates $[4,5]$. The current guidelines recommend RFCA as a preferred therapy in patients with symptomatic OTVAs [6]. However, structural complexity, anatomic variation, and proximity to the conduction system and coronary vasculature serve as the biggest obstacles to successful ablation. Therefore, accurate knowledge of the regional anatomy of these overlapping structures is essential

*Correspondence: kyoungmin.park@samsung.com

Division of Cardiology, Department of Medicine, Samsung Medical Center, Sungkyunkwan University School of Medicine, Seoul, Republic of Korea for the effective and safe ablation of OTVAs. Although three-dimensional (3D) mapping with fluoroscopy is often sufficient for guiding catheters, more detailed anatomic information is needed in many cases, especially in the left ventricular outflow tract (LVOT).

Since intracardiac echocardiography (ICE) was introduced in the 1980s, technological advances have continued, making it an integral part of various cardiac interventions [7]. In the field of electrophysiology, ICE allows accurate visualization of cardiac structures and continuous monitoring of catheter position, with integration of real-time images and electroanatomic mapping (EAM) $[8,9]$. In OTVAs, ICE has played an invaluable role in precisely defining the anatomic shell of OTs beyond fluoroscopy and in guiding catheter manipulation in relation to critical structures like the conduction system and coronary arteries. These roles could contribute to improving clinical outcomes and reducing complications $[10,11]$. From this point of view, we provide original author(s) and the source, provide a link to the Creative Commons licence, and indicate if changes were made. The images or other third party material in this article are included in the article's Creative Commons licence, unless indicated otherwise in a credit line to the material. If material is not included in the article's Creative Commons licence and your intended use is not permitted by statutory regulation or exceeds the permitted use, you will need to obtain permission directly from the copyright holder. To view a copy of this licence, visit http://creativecommons.org/licenses/by/4.0/. 
detailed information on comprehensive application of ICE for catheter ablation of OTVAs through this review article.

\section{Introduction of the ICE system}

The current ICE catheter used in electrophysiologic procedures consists of a 64-element, phased array transducer capable of high-resolution 2D and full Doppler/color flow imaging [12]. The ICE probe provides a 90-degree sector image perpendicular to the long axis of the catheter with tissue penetration of 15 to $18 \mathrm{~cm}$. The currently available ICE catheters in South Korea are the SOUNDSTAR ${ }^{\circledR} /$ ACUSON AcuNav $^{\mathrm{TM}}$ (Biosense Webster, Inc., Diamond Bar, CA) and ViewFlex ${ }^{\mathrm{TM}}$ Xtra (Abbott, Inc., Chicago, IL). Both ICE catheters can steer the catheter tip bidirectionally in two planes (antero-posterior and right-left) by manipulating the control handle [12]. The
ICE image is conventionally displayed in the orientation where the right side of the screen is superior anatomically and the downside of the screen indicates a far field from the transducer. The comparison of ICE catheters is summarized in Table 1.

\section{ICE views of the OT}

There was no standard view for ICE, as there are for transthoracic echocardiography and transesophageal echocardiography. However, some viewpoints can be useful in assessing the anatomy of the OTs. An ICE catheter can be easily advanced through the femoral vein into the right atrium (RA) without fluoroscopic guidance by an experienced operator. The ICE study is usually initiated with the "home view" acquired from the ICE catheter positioned in the mid-RA facing tricuspid valve (TV) (Fig. 1a). This view allows superb visualization of the TV

Table 1 Comparison of currently available ICE catheters in South Korea

\begin{tabular}{|c|c|c|c|}
\hline ICE catheter & SOUNDSTAR $^{\circledR}$ & AcuNav $^{\mathrm{TM}}$ & ViewFlex ${ }^{\mathrm{TM}}$ Xtra \\
\hline Manufacture & Biosense Webster & Biosense Webster & Abbott \\
\hline Transducer & 64-element vector phased array & 64-element vector phased array & 64-element vector phased array \\
\hline Frequency, MHz & $4-10$ & $4-10$ & $3-9$ \\
\hline Catheter size, F & 8 or 10 & 8 or 10 & 9 \\
\hline Length, cm & 90 & 90 & 90 \\
\hline Penetration depth, cm & 15 & 15 & 18 \\
\hline Tip deflection & Four directions (A/P/R/L) & Four directions (A/P/R/L) & Four directions (A/P/R/L) \\
\hline Deflection angle & $160^{\circ}$ & $160^{\circ}$ & $120^{\circ}$ \\
\hline Locking system & Manual & Manual & Auto \\
\hline Mode & 2D, M-mode, PW, CW, Color, Tissue Doppler ${ }^{*}$ & 2D, M-mode, PW, CW, Color, Tissue Doppler & 2D, M-mode, PW, CW, Color \\
\hline 3D mapping & Available & Not available & Not available \\
\hline System & Siemens or GE & Siemens or GE & Zonare or Philips \\
\hline Sterile sleeve & Need & Need & No need \\
\hline
\end{tabular}

ICE intracardiac echocardiography, $A$ anterior, $P$ posterior, $R$ right, $L$ left, $2 \mathrm{D}$, two-dimensional, $P W$ pulse wave, $C W$ continuous wave

* SIEMENS is an option

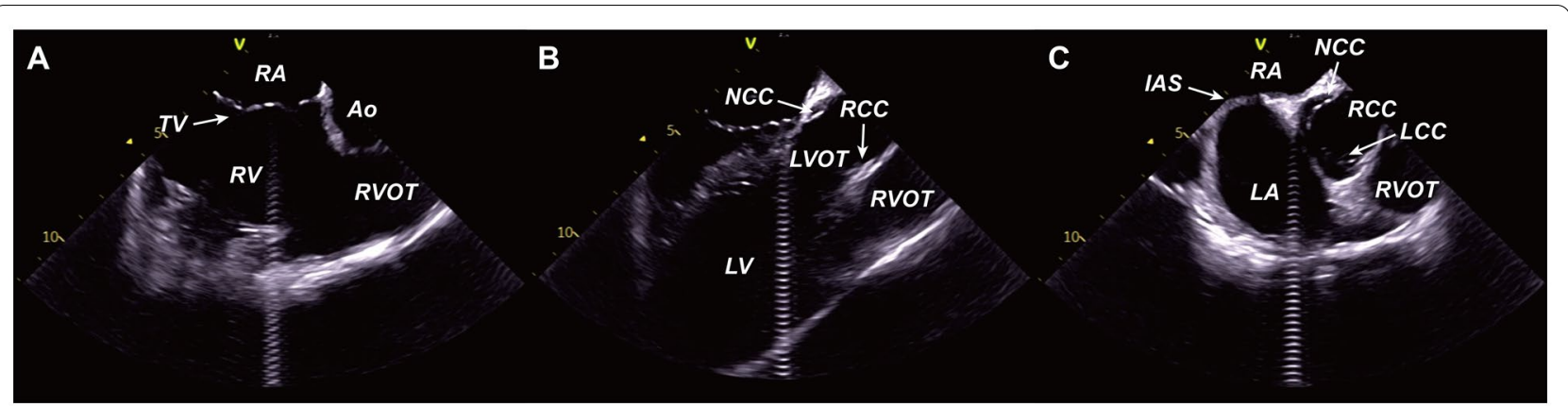

Fig. 1 Assessment of the ventricular outflow tracts from the right atrium. A. Home view. B. RVOT and LVOT view. C. Aortic valve short-axis view in RA. RVOT, right ventricular outflow tract; LVOT, left ventricular outflow tract; RA, right atrium; RV, right ventricle; TV, tricuspid valve; Ao, aortic root; RCC; right coronary cusp; LCC; left coronary cusp; NCC, non-coronary cusp; LV, left ventricle; IAS, interatrial septum; LA, left atrium 
and right ventricular (RV) inflow tract. A comprehensive evaluation of the OTs can be conducted in the RA and RV.

\section{From the RA}

The catheter is rotated clockwise with a neutral position from the home view to visualize the right ventricular outflow tract (RVOT) in the RA (Fig. 1a). In this view, the RVOT is seen just below the aortic root. The anterior (or septal) TV leaflet is visualized at 3 o'clock and is closed to the aortic root opposing the posterior leaflet at 9 o'clock.

From the RVOT view, the slightly clockwise rotation of the catheter will reveal the LVOT and aortic root in the long axis (Fig. 1b). On the other hand, the pulmonic valve (PV) will be shown in the short axis just behind the aortic valve (AV) since the OTs intersect at their origins on the disparate axis. In this view, the non-coronary cusp (NCC) is adjacent to the RA and TV in a close relationship with the parahisian region, whereas the opposite cusp is the right coronary cusp (RCC) close to the RVOT infundibulum. Further clockwise rotation facing the posterior and leftward side of the heart visualizes the LV cavity, anterior mitral leaflet (AML), and left atrium.

A short-axis view of the LVOT in the RA can be obtained by combining leftward control handle movement and clockwise rotation of the catheter. This view provides an excellent transverse image of the AV with a typical trileaflet appearance (Fig. 1c).

\section{From the RV}

From the home view, the curved catheter can be advanced across the TV with combined anterior and leftward movements of the control handle. Once the catheter tip passes through the TV, a slight release of the anterior curve will enable the catheter to advance further into the RV. In this catheter location, the RVOT and PV are viewed clearly in the long axis with the more distal part of the main pulmonary artery (Fig. 2a). The pulmonary cusp closest to the AV is the left pulmonary cusp (LPC), while the anterior pulmonary cusp (APC) is observed on the opposite side. Further advancement of the catheter allows visualization of the main pulmonary artery bifurcation.

A transverse view of the LVOT can be obtained inside the RV cavity as well as in the RA. First, the anteriorly curved catheter is advanced gently into the RV, and then the anterior deflection is released. By rotating the catheter clockwise, the transducer beam will move from the inferior RV to the LV cavity. The interventricular septum (Fig. 2b), posteromedial papillary muscle (Fig. 2c), anterolateral papillary muscle, and LV basal structures such as the AML and aorto-mitral continuity (AMC) are observed sequentially as the catheter rotates (Fig. 2d and e). With further clockwise rotation of the catheter from the view of the LV base, a transverse section of the aortic root comes into view with a typical MercedesBenz symbol (Fig. 2f). The cusp visualized nearest to the transducer is the RCC. The left coronary cusp (LCC) and NCC are observed in a clockwise manner from the RCC. From this view, the ostia of the left (Fig. 2g) and right coronary arteries (Fig. 2i) can be identified. In addition, careful counterclockwise rotation of the catheter allows for tracking of the left anterior descending artery (LAD) (Fig. 2H). On the other side, tracking of the right coronary artery (RCA) can be achieved from the RVOT looking up toward the aorta on the long axis. The RCA is observed just below the RCC.

\section{Guidance of OTVA ablation using ICE}

The shape, length, and size of the pulmonary infundibulum vary from patient to patient and are independent of RV size $[13,14]$. Therefore, the precise anatomic boundaries of the RVOT cannot be obtained by fluoroscopy alone, resulting in incomplete mapping of the SOO or damage to the thin RV free wall. This can be prevented by delineating the anatomic shell of the RVOT with the ICE before EAM (Fig. 3). The long- and short-axis views of the RVOT provide accurate boundaries of the supra- and infra-valvular regions and pulmonary semilunar leaflet. The LAD lies near the LPC and APC. It was reported that catheter ablation in the RVOT was associated with occlusion of LAD [15]. Additionally, severe pulmonary valve insufficiency with moderate proximal pulmonary artery stenosis was reported following RFCA ablation guided by EAM [16]. Complication during RVOT ablation is rare $[4,5]$ and is hindered by ICE assessment of the precise regional anatomy with continuous monitoring of the catheter position in critical structures such as valves and coronary arteries.

The rare but most serious complication during LVOT ablation is coronary artery injury. A recent systematic review article including 43 studies has reported three cases of coronary injury during LVOT and SoV ablation [17]. Although coronary angiography is usually performed before ablation, it is difficult to accurately determine the relationship between the actual positions of the catheter and the coronary artery during the entire cardiac cycle using fluoroscopy. Furthermore, aortic valve injuries during catheter ablation for VAs are rare but possible [18]. The short-axis view in the RV provides an accurate location of the ostium and course of the coronary arteries, as well as an excellent transverse view of the AV. Ablation at least $1 \mathrm{~cm}$ from the coronary ostium is generally safe [6]. Therefore, ICE use can overcome the limitations of fluoroscopy-based ablations, allowing for 


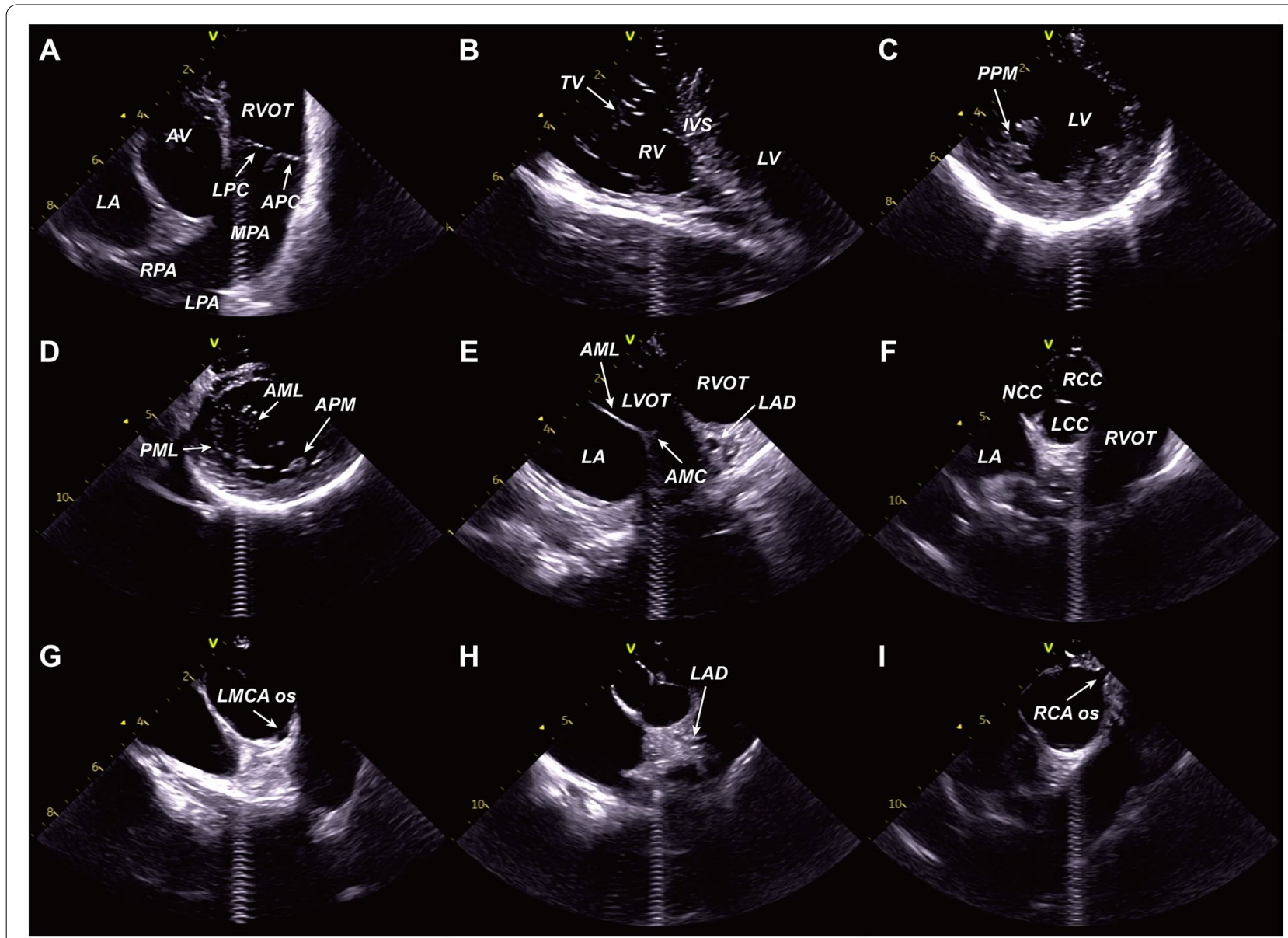

Fig. 2 Assessment of the ventricular outflow tracts from the right ventricle. A. RVOT and pulmonary artery. B. RV, LV, and interventricular septum (IVS). C. Posteromedial papillary muscle (PPM). D. Mitral leaflets and anterolateral papillary muscle (APM). E. Aorto-mitral continuity (AMC). F. Three aortic valve (AV) cusps. G. Left main coronary artery (LMCA) ostium (OS). H. Tracking of the left anterior descending artery (LAD). I. Right coronary artery (RCA) ostium. RVOT, right ventricular outflow tract; LPC, left pulmonary cusp; APC, anterior pulmonary cusp; MPA, main pulmonary artery; $R P A$, right pulmonary artery; $L P A$, left pulmonary artery; LA, left atrium; TV, tricuspid valve; RV, right ventricle; LV, left ventricle; AML, anterior mitral leaflet; PML, posterior mitral leaflet; RCC; right coronary cusp; LCC; left coronary cusp; NCC, non-coronary cusp

real-time visualization of the ablation catheter considering its proximity to the coronary artery.

ICE use also enables continuous monitoring of catheter-tissue contact, enabling effective energy delivery to the arrhythmogenic substrate. In addition, lesion formation can be identified as increased echogenicity and tissue thickness during ablation (Fig. 4) [19]. For these reasons, the utilization of ICE will enhance the effectiveness of catheter ablation.

The CARTO ${ }^{\circledR}$ 3D mapping system with the CARTOSOUND ${ }^{\circledR}$ module (Biosense Webster) can superimpose EAM on ICE-generated 3D images. There are several advantages that can be obtained by integrating ICE 3D images with EAM. First, precise delineation of the anatomic contour of the OTs using ICE enables more meticulous EAM that is essential to find SOO. Without clear anatomical boundaries, locations that are hard to reach with a mapping catheter can be omitted. However, acquisition of the anatomic shell of OT using ICE before EAM can guide EAM without missing spots. Second, the integration of 3D ICE images with EAM is particularly beneficial in mapping aortic SoV and coronary arteries that are not well visualized by fluoroscopy and EAM. When LVOT VA is suspected, the location of the aortic cusps and ostium and proximal course of the coronary arteries is initially delineated using ICE. Then, this anatomic information can be added to the 3D EAM to ensure a safe distance from coronary arteries without the need for a coronary angiogram or preprocedural imaging such as cardiac computed tomography and magnetic resonance imaging. Therefore, it will enhance the ability to map and 


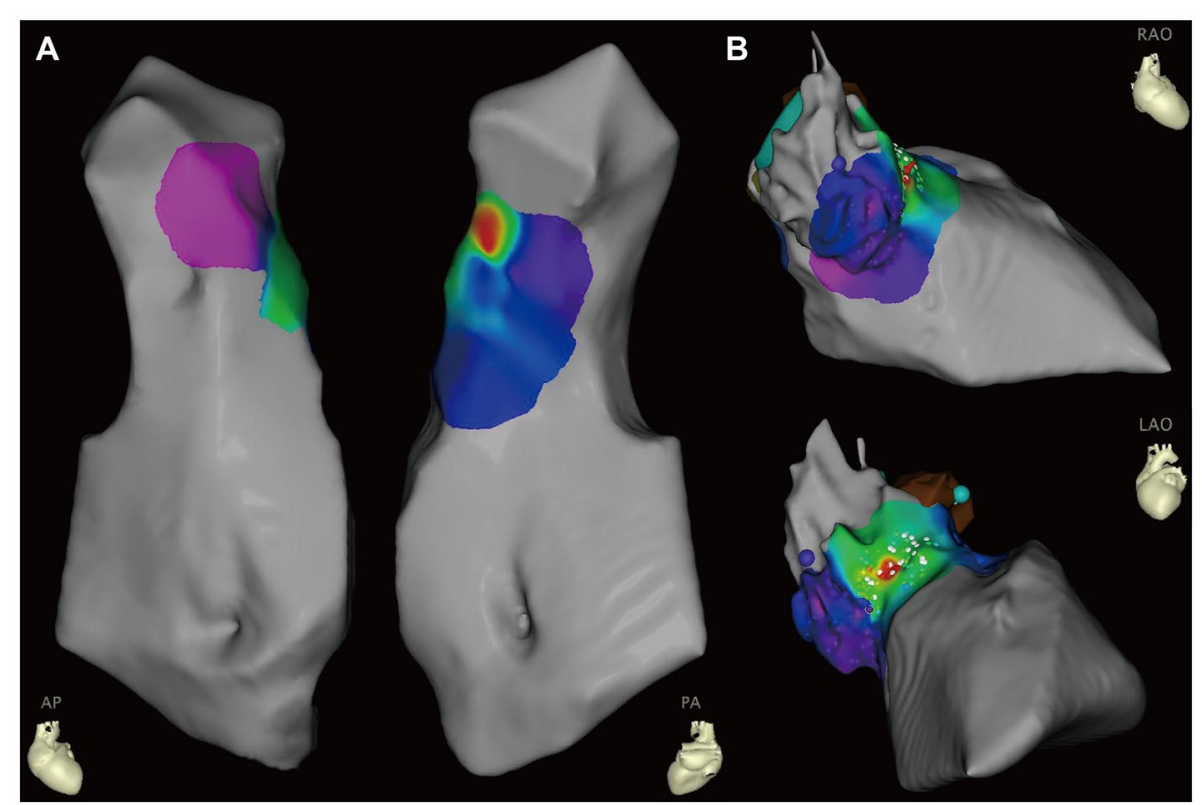

Fig. 3. Three-dimensional electroanatomical mapping of RVOT and LVOT incorporated with anatomic shell mapping using intracardiac echocardiography. A. Three-dimensional (3D) mapping of RVOT presented with antero-posterior view (left) and postero-anterior view (right) view. B. 3D mapping of LVOT presented with right anterior oblique (top) and left anterior oblique (bottom) view

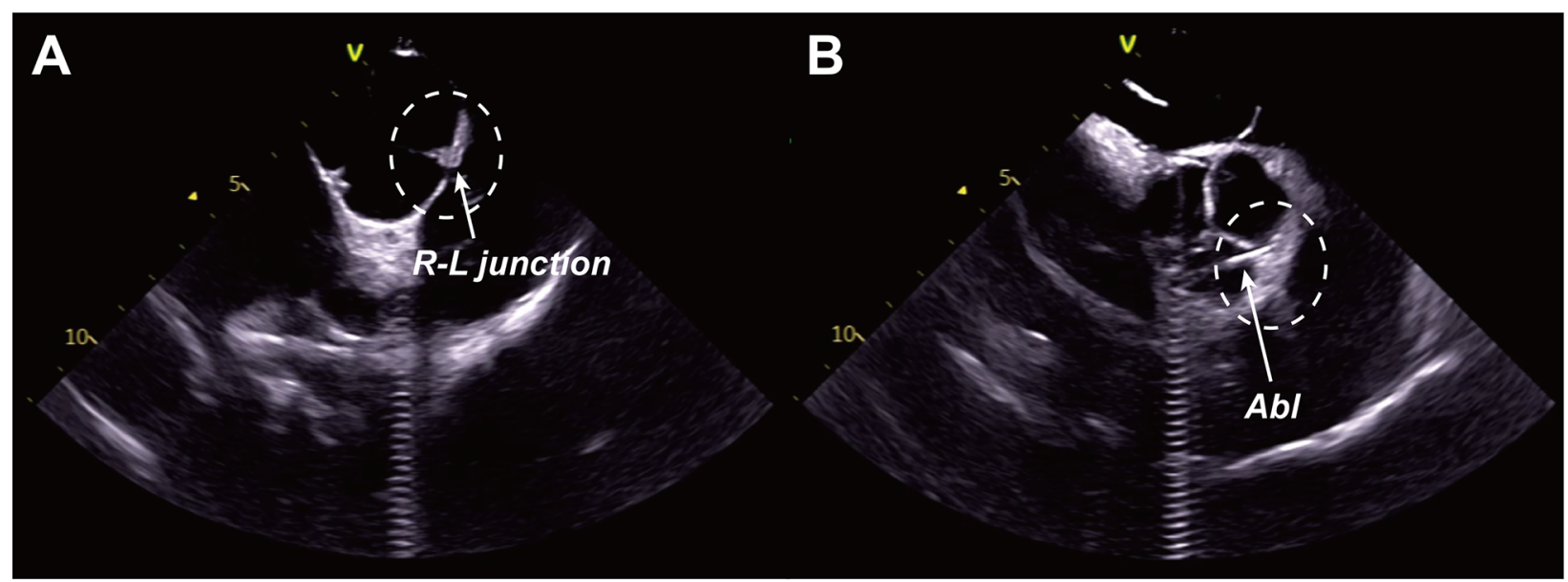

Fig. 4 Assessment of effective lesion formation using intracardiac echocardiography. A. Before ablation. B. During ablation at RCC-LCC junction (dashed line). Increased echogenicity was observed during ablation compared with before ablation. R-L, right coronary cusp-left coronary cusp; Abl, ablation catheter

determine the $\mathrm{SOO}$ of arrhythmias and reduce radiation exposure.

\section{Conclusions}

Understanding the complex anatomic relationship of OTs and accurate localization of critical structures like coronary arteries is essential to obtain the optimal results. ICE utilization with or without integration with electroanatomic mapping provides high-quality real-time visualization of cardiac structures and continuous monitoring of catheter location with ablation lesion formation, resulting in improved clinical outcomes of OTVA ablation. Randomized studies to investigate the efficacy and safety of ICE use in OTVA ablation are warranted. 


\begin{abstract}
Abbreviations
2D: Two-dimensional; 3D: Three-dimensional; Abl: Ablation catheter; AMC: Aorto-mitral continuity; AML: Anterior mitral leaflet; Ao: Aortic root; APC: Anterior pulmonary cusp; AV: Aortic valve; EAM: Electroanatomic mapping; IAS: Interatrial septum; ICE: Intracardiac echocardiography; LA: Left atrium; LAD: Left anterior descending; LAO: Left anterior oblique; LCC: Left coronary cusp; LPA: Left pulmonary artery; LPC: Left pulmonary cusp; LV: Left ventricle; LVOT: Left ventricular outflow tract; MPA: Main pulmonary artery; NCC: Non-coronary cusp; OT: Outflow tract; PML: Posterior mitral leaflet; PV: Pulmonic valve; RA Right atrium; RAO: Right anterior oblique; RCA: Right coronary artery; RCC : Right coronary cusp; RFCA: Radiofrequency catheter ablation; RPA: Right pulmonary artery; RV: Right ventricle; RVOT: Right ventricular outflow tract; SO: Site of origin; SoV: Sinus of valsalva; TV: Tricuspid valve; VAs: Ventricular arrhythmias.
\end{abstract}

\section{Authors' contributions}

Choi J.H. collected data and wrote a manuscript. Park K.M. reviewed and revised manuscript.

\section{Availability of data and materials}

The datasets used and/or analyzed during the current study are available from the corresponding author on reasonable request.

\section{Declarations}

\section{Ethical approval and consent to participate}

Not applicable.

\section{Consent for publication}

Written informed consent for publication of their clinical details and/or clinical images was obtained from the patient/parent/guardian/ relative of the patient.

\section{Competing interests}

The authors declare that they have no competing interests.

Received: 1 July 2021 Accepted: 20 October 2021

Published online: 02 February 2022

\section{References}

1. Yamada T. Idiopathic ventricular arrhythmias: relevance to the anatomy, diagnosis and treatment. J Cardiol. 2016;68(6):463-71.

2. Maury P, Rollin A, Mondoly P, Duparc A. Management of outflow tract ventricular arrhythmias. Curr Opin Cardiol. 2015:30(1):50-7.

3. Stevenson WG, Soejima K. Catheter ablation for ventricular tachycardia. Circulation. 2007;115(21):2750-60.

4. Ling Z, Liu Z, Su L, Zipunnikov V, Wu J, Du H, et al. Radiofrequency ablation versus antiarrhythmic medication for treatment of ventricular premature beats from the right ventricular outflow tract: prospective randomized study. Circ Arrhythm Electrophysiol. 2014;7(2):237-43.

5. Latchamsetty R, Yokokawa M, Morady F, Kim HM, Mathew S, Tilz R, et al. Multicenter outcomes for catheter ablation of idiopathic premature ventricular complexes. JACC Clin Electrophysiol. 2015;1(3):116-23.
6. Cronin EM, Bogun FM, Maury P, Peichl P, Chen M, Namboodiri N, et al. 2019 HRS/EHRA/APHRS/LAHRS expert consensus statement on catheter ablation of ventricular arrhythmias. Europace. 2019;21(8):1143-4.

7. Jongbloed MRM. Clinical applications of intracardiac echocardiography in interventional procedures. Heart. 2005;91(7):981-90.

8. Nakamura K, Naito S, Kaseno K, Nakatani Y, Sasaki T, Anjo N, et al. Integration of intracardiac echocardiography and computed tomography during atrial fibrillation ablation: combining ultrasound contours obtained from the right atrium and ventricular outflow tract. Int J Cardiol. 2017;228:677-86

9. Marrouche NF, Martin DO, Wazni O, Gillinov AM, Klein A, Bhargava M, et al. Phased-array intracardiac echocardiography monitoring during pulmonary vein isolation in patients with atrial fibrillation: impact on outcome and complications. Circulation. 2003;107(21):2710-6.

10. Jongbloed MR, Bax JJ, van der Burg AE, Van der Wall EE, Schalij MJ. Radiofrequency catheter ablation of ventricular tachycardia guided by intracardiac echocardiography. Eur J Echocardiogr. 2004;5(1):34-40.

11. Lamberti F, Calo L, Pandozi C, Castro A, Loricchio ML, Boggi A, et al. Radiofrequency catheter ablation of idiopathic left ventricular outflow tract tachycardia: utility of intracardiac echocardiography. J Cardiovasc Electrophysiol. 2001;12(5):529-35.

12. Alkhouli M, Hijazi ZM, Holmes DR Jr, Rihal CS, Wiegers SE. Intracardiac Echocardiography in Structural Heart Disease Interventions. JACC Cardiovasc Interv. 2018;11(21):2133-47.

13. Chung R, Taylor AM. Imaging for preintervention planning: transcatheter pulmonary valve therapy. Circ Cardiovasc Imaging. 2014;7(1):182-9.

14. Saremi F, Ho SY, Cabrera JA, Sanchez-Quintana D. Right ventricular outflow tract imaging with CT and MRI: Part 1. Morphology AJR Am J Roentgenol. 2013;200(1):W39-50.

15. Benhayon D, Nof E, Chik WW, Marchlinski F. Catheter Ablation in the Right Ventricular Outflow Tract Associated With Occlusion of Left Anterior Descending Coronary Artery. J Cardiovasc Electrophysiol. 2017:28(3):347-50.

16. Bansal N, Kobayashi D, Karpawich PP. Pulmonary damage following right ventricular outflow tachycardia ablation in a child: When electroanatomical mapping isn't good enough. Pacing Clin Electrophysiol. 2018:41(5):561-5.

17. Pothineni NV, Kancharla K, Katoor AJ, Shanta G, Paydak H, Kapa S, et al. Coronary artery injury related to catheter ablation of cardiac arrhythmias: a systematic review. J Cardiovasc Electrophysiol. 2019;30(1):92-101.

18. Plachinski SJ, Salman SS, Carey J, Flanagan CM, Novalija J, Pagel PS, et al. latrogenic Aortic Insufficiency After Radiofrequency Ablation of the Left Ventricular Outflow Tract. J Cardiothorac Vasc Anesth. 2021.

19. Callans DJ, Ren JF, Narula N, Michele J, Marchlinski FE, Dillon SM. Effects of linear, irrigated-tip radiofrequency ablation in porcine healed anterior infarction. J Cardiovasc Electrophysiol. 2001;12(9):1037-42.

\section{Publisher's Note}

Springer Nature remains neutral with regard to jurisdictional claims in published maps and institutional affiliations.

Ready to submit your research? Choose BMC and benefit from:

- fast, convenient online submission

- thorough peer review by experienced researchers in your field

- rapid publication on acceptance

- support for research data, including large and complex data types

- gold Open Access which fosters wider collaboration and increased citations

- maximum visibility for your research: over 100M website views per year

At BMC, research is always in progress.

Learn more biomedcentral.com/submissions 